\title{
Synchronous gesture manipulation for collaboration and coordination of co-located Business Process Modelling
}

\author{
Brenda Scholtz ${ }^{a}$, André Calitz ${ }^{a}$, Irene Snyman ${ }^{b}$ \\ ${ }^{a}$ Computing Sciences Department, Nelson Mandela Metropolitan University, South Africa \\ ${ }^{b}$ SYSPRO, Port Elizabeth, South Africa
}

\begin{abstract}
The purpose of this study was to investigate approaches (techniques and technologies) for the coordination of collaborative tasks using synchronous gesture manipulation. Business Process Modelling (BPM) tasks are often performed in teams of modellers who need to collaborate with each other in order to coordinate and integrate their individual contributions into the various process models in a co-located environment.

These collaborative BPM tasks were used as a case study in order to develop the artifact (the BPM-Touch approach) as a proof of concept. The BPM-Touch approach allows for the coordination and collaboration of BPM tasks in co-located modelling teams using synchronous gesture manipulation approaches. The Design Science Research (DSR) methodology was used and several cycles of developing and evaluating the artifact took place.

This paper reports on the last cycle and set of evaluations. The proposed approach was implemented in a BPM software package in order to provide empirical validation. Usability evaluations of the software were undertaken with both students and BPM professionals as participants. The empirical results of the evaluations revealed that the participants found the approach to be effective and rated the usability and satisfaction of the collaboration and gesture manipulation aspects of the software positively.
\end{abstract}

Keywords: Business process modelling, gesture manipulation, coordination, collaborative BPM

\section{Categories:}

H.5.2 [User Interfaces]: Evaluation/methodology; Input devices and strategy.

K.3.1 [Group and Organization Interfaces]: Collaborative computing; Synchronous interaction.

\section{Email:}

Brenda Scholtz brenda.scholtz@nmmu.ac.za (CORRESPONDING),

André Calitz andre.calitz@nmmu.ac.za,

Irene Snyman irene.snyman@syspro.za.com
Article history:

Received: 27 Aug 2014

Accepted: 16 Jul 2015

Available online: 10 Dec 2015

\section{INTRODUCTION}

Collaboration and coordination are key factors that are required in order to support interaction in a team environment (Denise, 1999). Several styles have been proposed for working and interacting

Scholtz, B., Calitz, A., \& Snyman, I. (2015). Synchronous gesture manipulation for collaboration and coordination of co-located Business Process Modelling. South African Computer Journal 57, 90-112. http://dx.doi.org/10.18489/ sacj.v0i57.269

Copyright (C) the author(s); published under a Creative Commons NonCommercial 4.0 License (CC BY-NC 4.0). $S A C J$ is a publication of the South African Institute of Computer Scientists and Information Technologists. ISSN 1015-7999 (print) ISSN 2313-7835 (online). 
in a co-located, collaborative environment (Isenberg, Fisher, Morris, Inkpen \& Czerwinski, 2010). These styles vary from a close collaboration style where discussion takes place around a subject to a loose collaboration style where one participant is working and the other is watching and not engaged in the work at all. Studies (Oleksik, Milic-Frayling \& Jones, 2014; Morris, Huang, Paepcke \& Winograd, 2006; Hinckley, 2003; Hinckley, Ramos, Guimbretiere, Baudisch \& Smith, 2004) have shown that a useful and efficient platform for performing collaborative tasks are touch technologies incorporating synchronous gestures. The rapid growth of Tablet PCs (PC Tech Staff, 2014) has facilitated the emergence of techniques for improving the effectiveness of collaborative tasks using these technologies (Hinckley, 2003; Hinckley et al., 2004).

The use of synchronous gestures is one such technique and refers to particular patterns of activities where the outcomes of these patterns change when the activities take place simultaneously. Dynamic display tiling is a technique that incorporates synchronous gestures and allows the displays on different Tablet PCs to be shared by bumping the devices against each other (Hinckley, 2003). Another synchronous gesture technique that has been used is stitching, an an approach that uses a pen to connect mobile devices and create a shared working area for collaboration (Hinckley et al., 2004). The use of touch and synchronous gestures has opened up opportunities for research related to emerging user practices and experiences related to co-located collaborative tasks.

One area where collaborative tasks are performed is in Business Process Modelling (BPM) projects in organisations, as well as in BPM courses in Higher Education Institutions (HEIs). BPM is often performed as a collaborative activity that needs to be carried out in a team environment. One of the benefits of this collaboration is the reduced individual workload for modellers (Forster, Pinggera \& Weber, 2012). The number of BPM initiatives has increased significantly in recent years and BPM tasks are increasing in frequency in organisations that need to analyse and optimise their business processes (Scheer \& Brabänder, 2010). Research conducted by Global Industry Analysts, suggests that the global market for BPM will reach $\$ 5.3$ billion by 2017 (Gaskell, 2012). The demand for and predominance of BPM has led to a need for quality BPM skills. Whilst these skills are highly sought after, they are not easily available globally and this is also true for developing countries such as South Africa (Flügel, Seymour \& van der Merwe, 2014). The demand for BPM skills has resulted in the incorporation of BPM as a recommended learning objective of the IS Curriculum (Topi et al., 2010), and has pressurised HEIs globally to introduce BPM courses into their IS curricula (Bandara et al., 2010; Seethamraju, 2012). HEIs in South Africa are also following this trend (Flügel et al., 2014; Scholtz, Calitz \& Snyman, 2013; Van Greunen, Van Der Merwe \& Kotze, 2010).

The collaborative activities of BPM can be referred to as Collaborative Business Process Modelling (CBPM). Benefits of CBPM are the improved readability, accuracy and quality of process models (Barjis, 2011). Several studies (Flügel et al., 2014; Scholtz et al., 2013; Van Greunen et al., 2010; Bandara et al., 2010; Recker, 2010b) have investigated the challenges of BPM tools in education and in industry. However, at the time of writing, only a limited number of studies (Forster et al., 2012; Dollmann, Houy, Fettke \& Loos, 2011) related to the collaboration and coordination of the task of creating process models could be found and none of these investigate the use of synchronous gestures and touch technologies for collaborative tasks.

This paper addresses a gap in the field of collaborative research, particularly with regards to 
gesture manipulation approaches. The main aim of the paper is to investigate collaboration and coordination approaches using synchronous gesture manipulation on mobile device technologies. CBPM is used as a case study and a prototype was developed and evaluated for usability as a proof of concept. This study also aims to stimulate debate and future research in the field of gesture manipulation and the use of mobile devices for performing BPM tasks.

The paper is structured as follows: Section 2 discusses several theories and approaches for collaboration, coordination and synchronous gestures. Section 3 describes the research methods and theoretical model used in this empirical study as well as the design and evaluation of the BPM-Touch prototype. The results of the evaluation are analysed and reported on in Section 4. Several conclusions and recommendations for future research are made in the last section.

\section{COLLABORATION AND SYNCHRONOUS GESTURES}

In order to support interaction in a team or group setting, several factors need to be taken into consideration, namely collaboration, coordination and communication (Denise, 1999). Effective collaboration involves information sharing between and amongst group participants (Ellis, Gibbs \& Rein, 1991). It is important that group participants receive notifications of other participants' activities if it is deemed necessary as well as up-to-date displays of information. Coordination within a group of participants increases the effectiveness of the communication and collaboration factors (Denise, 1999). With coordination group conflict is reduced and repetition of actions and work within a group can be minimised (Morris et al., 2006; Denise, 1999). Coordination also involves notifying each part of the group how to act and when the right time would be to act. Interactions requiring explicit coordination between two or more users can promote an improved sense of cohesion in the group (Oleksik et al., 2014; Morris et al., 2006).

The challenges of collaborative, co-located environments with small teams of people can be overcome by using technologies such as touch devices (Scott, Carpendale \& Inkpen, 2004). Touch input has also been shown to require less concentration from users than mouse input (Hornecker, Marshall, Dalton \& Rogers, 2008). One type of technology that supports touch input is the multitouch surface. The multi-touch is a collaborative technology that allows co-located individuals to collaborate, while interacting together on one surface simultaneously using their fingers as a means of touch input instead of a keyboard or mouse (Morris et al., 2006; Hornecker et al., 2008; Kammer, Wojdziak, Keck, Groh \& Taranko, 2010; Hunter \& Maes, 2008). Using this type of input is a direct way of interacting with the screen and it has been reported to feel like a more natural approach. Hornecker et al. (2008) studied different facets of awareness with multi-touch input and multi-mice input on multi-touch surfaces and discovered that higher levels of awareness were achieved for multi-touch input. Multi-touch technology can also provide a more intuitive means of interaction than keyboard input. On a multi-touch surface several individuals can interact together due to the ability of simultaneous input/interaction supported. This is advantageous as all the individuals can take part in the activities and the discussion while interacting on the surface. Individuals do not have to sit and watch one person working and everyone's input can be taken into account. Using a multi-touch surface in a collaborative environment poses many benefits, such as being able to see 
other participants' body language, participants' pointing to objects on the surface and discussing the work at hand together (Clifton et al., 2011). Collaborative drawing and modelling can also be successfully achieved on a multi-touch surface (Morris et al., 2006; Hornecker et al., 2008; Kammer et al., 2010; Hunter \& Maes, 2008; Sams, Wesson \& Vogts, 2011).

Different ways of collaborating around a multi-touch surface exist and a study done by Isenberg et al. (2010) reported that when participants work in a co-located, collaborative environment around a multi-touch surface they could solve a complex task successfully. In their study they documented eight styles in which participants can collaborate within different activities (Figure 1). These styles range from close collaboration styles (a discussion about the subject matter) to loose collaboration styles (one participant working while the other participant is not engaged in the work at all). People do not collaborate in the same manner and different collaboration styles work differently to solve problems.

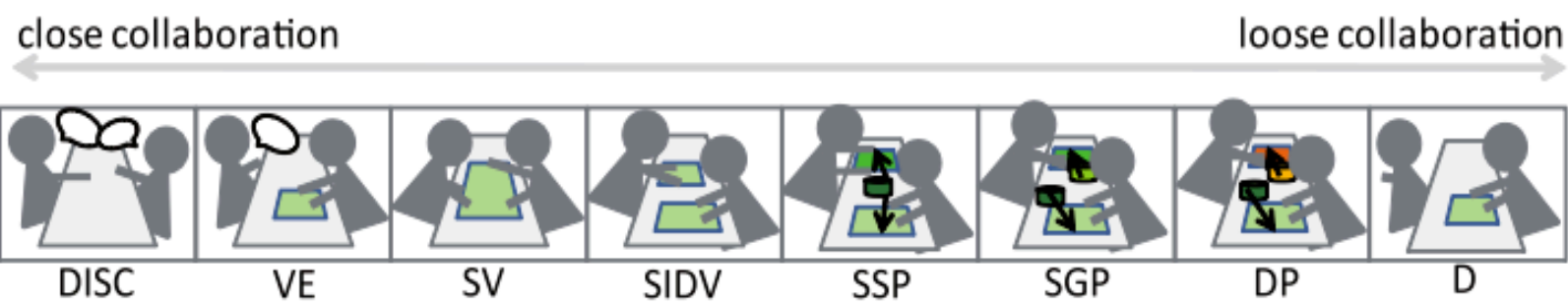

Figure 1: Collaboration styles around a multi-touch surface (Isenberg, Fisher, Morris, Inkpen \& Czerwinski, 2010)

The DISC collaboration style refers to a discussion between participants about the work at hand, VE refers to 'view engaged', meaning that one participant works while another participant watches and can make comments. SV indicates that participants share the 'same view' whilst working on a subject at hand; this could include from reading documents to moving objects around. SIDV is a close collaboration style and refers to the sharing of the 'same information at different views', meaning that participants interact with the same data but they each have their own copy of the data. SSP refers to the 'same specific problem', that relates to work being undertaken to solve a shared problem that has been clearly specified. In this case participants do not work from the same document but will read documents relating to the same problem from a shared set of documents. The SGP collaboration style refers to the 'same general problem', relating to participants working on the same problem but finding the data for the problem from different places and working from their own angle towards solving the problem. DP refers to 'different problems', in which case participants do not work together on solving the same piece of a problem but instead focus on solving different aspects of a problem. D refers to 'disengaged', in which case one participant interacts with the subject matter while the other participant does not take part at all.

The collaboration styles proposed by Isenberg et al. (2010) could also be considered in other types of touch technology besides the multi-touch. Another type of touch technology that has been used for drawing and modelling contexts as well as in education is the interactive whiteboard (IWB). 
The IWB allows the user to interact with the whiteboard instead of utilising a keyboard and mouse. IWBs also fall under the term touch technology as it detects the presence and position of touch input within an IWB display (G.-J. Hwang, Wu \& Kuo, 2013). Renger, Kolfschoten and de Vreede (2008) reported that IWBs are advantageous in a modelling environment as users can edit the model directly and this inspires participation and joint ownership.

However, in spite of the potential benefits of IWBs in both educational and organisational environments, they are extremely expensive and are generally immobile (Derizemlya, 2009). In educational environments IWBs are now being slowly replaced in popularity by Tablet PCs as they are more mobile, cost effective and versatile (Sheehy et al., 2005). Tablet PCs have also been shown to aid in collaboration and interaction between students and the teacher, as well as to promote interaction amongst and between students (Bonastre, Benavent \& Belmonte, 2006; Berry \& Hamilton, 2006). Laptops and mobile phones are rapidly being replaced by smartphones and Tablet PCs in organisations since the recent gain in momentum in the bring your own device (BYOD) movement by which employees take their personal mobile devices to work and use them to carry out their work activities (Technology Evaluation Centers, 2013). South Africa has followed the trend towards mobile devices and Tablet PC shipments into South Africa are predicted to expand at a compound annual growth rate of $13.5 \%$ from 2014 to a total 3.1 million units in 2018. The expectation is that the traditional notebook market will be much less healthy (PC Tech Staff, 2014).

Tablet PCs have been shown to be a useful and efficient platform for collaborative tasks, particularly for the use of synchronous gestures (Hinckley, 2003; Hinckley et al., 2004). Different devices give off different signals that are picked up by corresponding devices when they come into contact with each other. Dynamic display tiling is one way by which individuals can bring together the displays on different Tablet PCs (Hinckley, 2003). This is achieved by softly bumping a Tablet PC in an individual's hand against a still Tablet PC lying on a desk. When the individual bumps the Tablet PCs, one display is created and when a Tablet PC is picked up, it is detached from the joint display. Information can be shared among Tablet PCs by bumping the Tablet PCs against each other.

Data can also be sent from one Tablet PC to another by "pouring" the data to the other Tablet PC. The "pouring" of data is done by angling the Tablet PC containing the data downwards when bumping the other Tablet PC. The Tablet PCs can connect both horizontally and vertically. Another synchronous gesture developed is known as stitching (Hinckley et al., 2004). Stitching involves the use of a pen to connect two mobile devices (Figure 2). The gesture uses a pen and spans over several displays by starting the motion on one screen, skipping over the edges of the screen and ending on the screen of a separate device. In this way, a shared working area for collaboration is created.

Hand gestures using touch technology were successfully adopted in the CollabDraw software (Morris et al., 2006) and allowed users to collaboratively create diagrams and share pictures on a multi-touch, single display tabletop device. The participants in the CollabDraw study found the system easy to use and the gestures easy to learn. Another study (Oleksik et al., 2014) using touch and gestures but this time in a multi-device collaborative environment, reported that consistency and coordination of the touch commands across devices are important design criteria.

Whilst Tablet PCs have several benefits, they also have some limitations (Sheehy et al., 2005). The cost of tablet PCs is higher than the cost of laptops with comparable specifications. Tablet PCs 


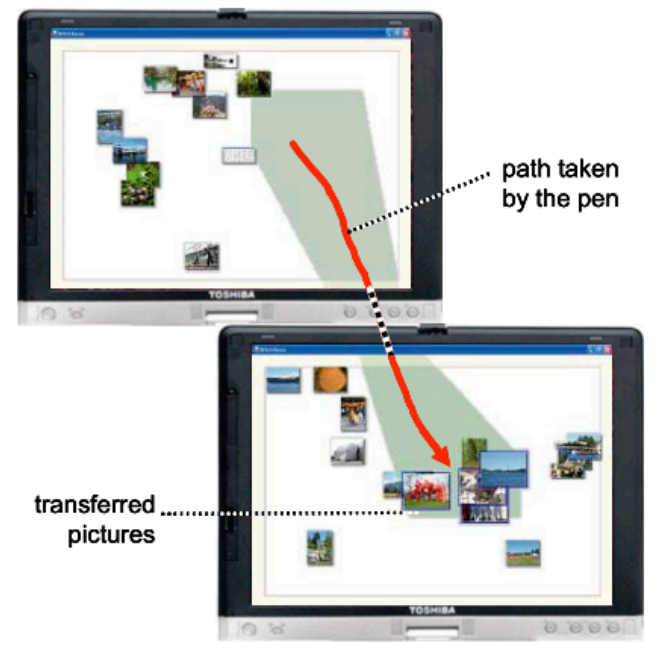

Figure 2: Sharing of photos between two tablets by stitching from one tablet to another (Hinckley, Ramos, Guimbretiere, Baudisch \& Smith, 2004)

also have low screen illumination and this makes it challenging to work with them outside in the light and they have a slow boot-up speed. Tablet PCs are also very time-consuming to set-up and slow networks limit the use of these devices (Bamforth, 2012). Small text needs to be avoided and the "fat finger" is a common problem with designing applications for touch (Apted \& Kay, 2008). The "fat finger" phenomenon is that everything represented on the surface must be large enough so that a fat finger can select the objects and manipulate them. If objects are not big enough, participants will find it difficult and frustrating to interact with and manipulate the objects on the surface.

\section{RESEARCH METHODOLOGY}

The research process included a literature review of collaborative techniques and technologies and a theoretical framework was derived. In order to gain an in-depth understanding of the properties and problems related to synchronous gesture manipulation, co-located Business Process Modelling (BPM) tasks requiring collaboration amongst team members were used as a suitable context of use and case study (Section 3.1). Once the requirements were determined a prototype (the artifact) was developed (Section 3.2). The proposed approach adopted the SIDV style and design guidelines recommended in other studies (Section 3.3). The evaluation process was carefully designed to make use of appropriate usability attributes and the participant sample consisted of students from a BPM course and BPM professionals (Section 3.4). 


\subsection{Context of use and case study}

Business Process Modelling (BPM) involves the human activity of creating a business process model that is an abstraction of a real-world business process as understood by the modeller (Mendling, 2007). The objectives of BPM have extended from simply configuring manual or repetitive tasks, to serve as training material for new employees and to create a common understanding of the interactions and dependencies among people, organisational operations, the systems they use and the information required to do their tasks effectively (Recker, 2010a). Recker (2010b) describes BPM as an essential cornerstone in the initial capturing and documentation of existing business processes, as well as in the specification of the redesigned, automated or amended business processes. A business process model consists of a network of business process diagrams that depict the number and sequence of activities in a process graphically. One notation for modelling business processes that has become the de facto standard for graphical process modelling is the Business Process Model and Notation (BPMN) that was developed by an industry consortium ${ }^{1}$ (Object Management Group, 2006). BPMN is widely supported by both free and commercial software modelling tools. In addition a large number of BPM consultants and BPM coaches are using BPMN as part of their offerings. Whilst BPMN is one of the most popular graphical modelling standards, several others exist, of which the Event-driven Process Chains (EPCs) (Scheer, 1998) and Unified Modelling Language (UML) activity diagrams (White, 2004) are two commonly used techniques. Throughout the business process management lifecycle, BPM tasks are critical, since they form the foundation of the understanding and restructuring of process activities, information flows and roles of participating individuals and applications for process optimisation (Mendling, 2007; Ko, Lee \& Wah Lee, 2009). A lack of flexible BPM tools to support the ever-changing nature of business processes has been reported (Van Greunen et al., 2010).

There are several advantages of collaborating in teams to perform BPM tasks by sharing large process models and diagrams (Scholtz et al., 2013; Forster et al., 2012). However, several problems related to collaborating, coordinating and integrating models have been reported by modellers (Scholtz et al., 2013; Forster et al., 2012). Process modellers working in teams often collaborate during BPM activities in the same location (co-located) and on the same models and therefore require approaches that can support the coordination of synchronous modelling tasks.

\subsection{Objectives}

The primary problem that this study addresses is the lack of empirically validated approaches related to the coordination of collaborative tasks using gesture manipulation. The main aim of the study is to propose and evaluate an approach for the synchronous collaboration of co-located tasks using gesture manipulation. The study uses co-located BPM modelling tasks as a case study (Section 3.1). The main research question addressed by this paper is therefore:

"Can a synchronous gesture manipulation approach be used for the effective collaboration and coordination of BPM tasks?"

\footnotetext{
${ }^{1}$ http://www.BPMI.org
} 
In order to answer this question several objectives were identified, namely:

1. Design an approach that allows for the synchronous collaboration and coordination of colocated BPM tasks using gesture manipulation;

2. Develop a prototype using a synchronous coordination approach and incorporating gesture input (the BPM-Touch); and

3. Evaluate the usability and collaboration of the prototype using the BPM-Touch.

The Design Science Research (DSR) methodology proposed by Johannesson and Perjons (2012) was used in the study. The DSR is an iterative approach that revolves around defining and identifying a problem that can be addressed by the development of an artifact. The DSR process documented by Johannesson and Perjons (2012) is similar to the one proposed by Peffers, Tuunanen, Rothenberger and Chatterjee (2007) and Hevner, March, Park and Ram (2004). The DSR framework recommended by Hevner (2007) consists of three cycles: the relevance, design and rigor cycles. The DSR was used in conjunction with a case study research approach using the case of collaboration in BPM in education and in BPM consulting. Multiple iterations of the design cycle were conducted and three versions of the artifact were produced and evaluated. Due to paper length constraints this paper only reports on the third iteration artifact and the related summative evaluations.

\subsection{The BPM-Touch Approach}

The collaboration styles described by Isenberg, et al. (Isenberg et al., 2010) can be extended to other touch technologies that are more cost-effective and mobile, such as the Tablet PC. In particular, the SIDV style is similar to the nature of the collaborative tasks performed by modellers working on a process model or set of models in a co-located environment. This is because modellers share the same information (the set of business process diagrams) and interact with these, but each modeller has their own copy/view of the diagrams. Synchronous gestures in mobile devices can therefore be used on these tablets to support the coordination and collaboration of tasks. The theories contributing to this approach are illustrated in Figure 3.

The goal in creating the BPM-Touch prototype and the set of gestures was to experiment and evaluate the techniques and technology proposed. ProcessCraft is a BPM application that was originally developed by a company called Tabtou using the Python programming language and runs on the Android platform (Tabtou, 2012). ProcessCraft is the first BPM tool that was designed with touch input and it runs on all contemporary operating systems. The software caters for the creation of BPMN models using a Tablet PC and collaboration is supported by enabling users to save the model and email it to a client or stakeholder. This is achieved by providing users with the opportunity to enter a 'presentation mode' in which the menus and background grid are removed in order to display the model in full screen. An image can then be generated and loaded into the user's email for distribution to the relevant clients or stakeholders.

The navigation of ProcessCraft includes the diagram canvas, symbol pallet, symbol menu, properties pane, help, presentation mode, intelli-menu, gestures and presentation mode (Tabtou, 2012). 


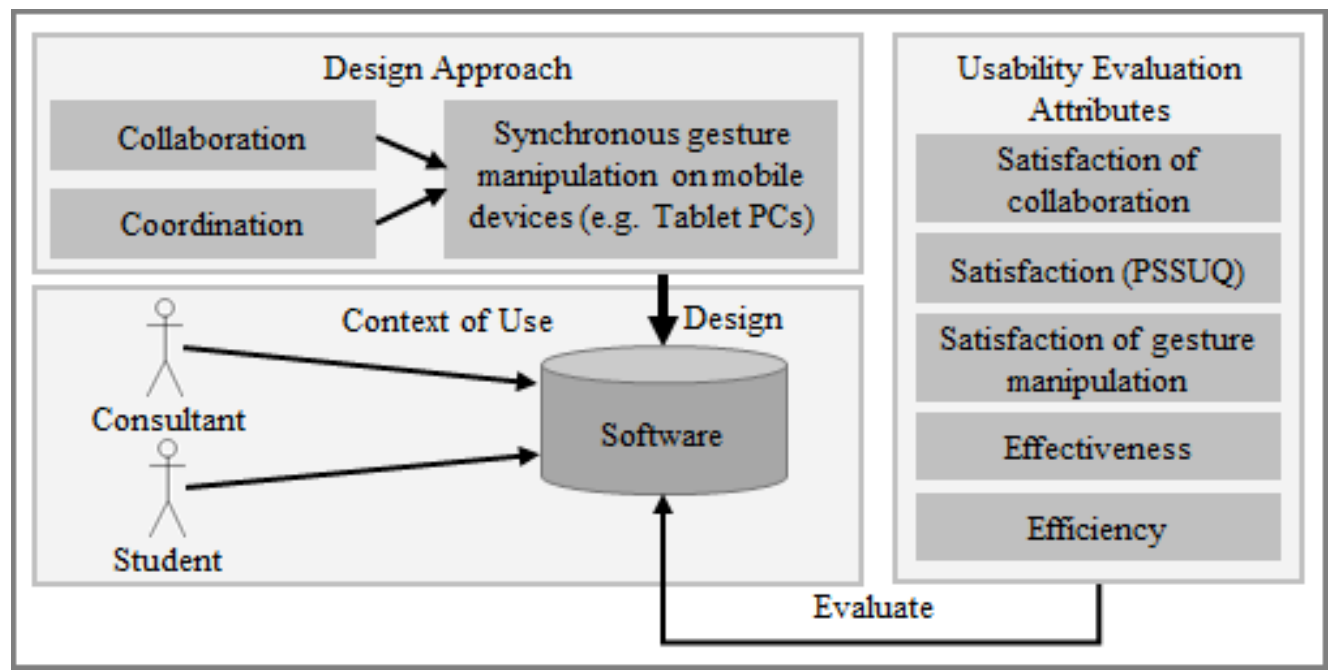

Figure 3: A synchronous gesture approach for collaborative tasks

The diagram canvas is a drawing board that carries on indefinitely, depending on the memory of your device. Users do not have to limit their model size to the screen size. The symbol pallet allows users to carry out a drag and hold gesture to pull the symbol from the symbol pallet onto the diagram canvas. The presentation mode provides users with a full-screen canvas on which the diagram can be navigated by the use of a drag gesture. Pinch (pinch two fingers together) and scale (drag two fingers apart) gestures are recognised by ProcessCraft and can be used to scale the canvas (Figure 4). This allows users to add more or less symbols into the viewing area of the canvas. A single finger drag can be used against the background of the drawing canvas and will result in the panning of the diagram in any desired direction on the infinite viewing canvas. Diagrams can be saved as a BPMN document, or shared as a BPMN 2.0 XML or PNG Graphic file.

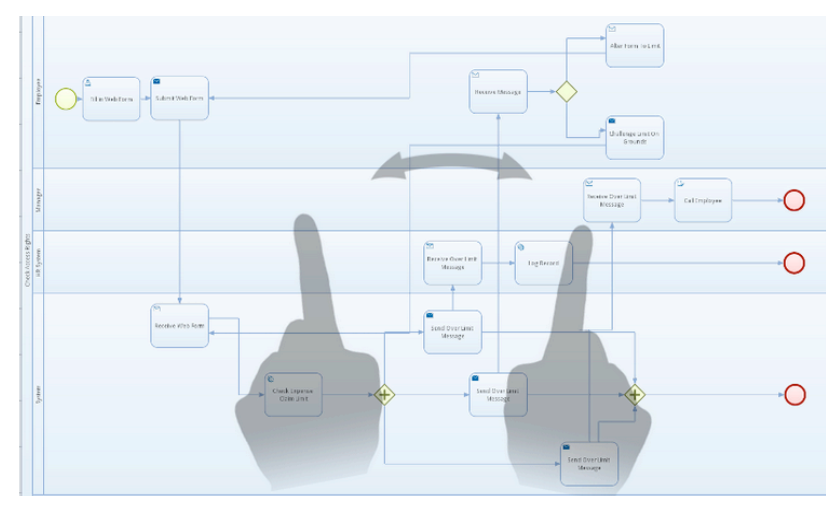

Figure 4: Pinch and scale gestures in BPMN (Tabtou, 2012) 
The ProcessCraft software was evaluated based on the knowledge gained from theoretical studies. The evaluation results showed that several requirements for synchronous CBPM were not satisfied, since it did not support effective collaboration and coordination amongst a small team of modellers. More specifically, the following requirements were not met:

- Support for the SIDV collaboration style (Isenberg et al., 2010), whereby changes made by one modeller on one Tablet PC update the models on all of the other connected Tablet PCs;

- Built-in client and server capabilities; and

- The software did not have a built-in locking mechanism.

Therefore the ProcessCraft software did not allow multiple users to work on a model in the same place and at the same time (co-located and synchronous). Modifications had to be made to the source code of ProcessCraft in order for the resulting BPM-Touch software solution to meet all of the requirements for a CBPM tool based on the BPM-Touch theoretical approach. A collaboration function was added to cater for a minimum of two users (one per tablet PC) and to allow for the synchronous coordination of models using the SIDV collaboration style. The new collaboration function can be activated by clicking on the collaboration button, and then one Tablet PC acts as a server and the other Tablet PCs can act as clients which can connect to the server wirelessly. Upon connection to the server, clients automatically "share" a screen with the server Tablet PC, whilst using their own Tablet PC.

In order to cater for the synchronous coordination of models between modellers, the source code was modified to allow for a locking mechanism (flag). A flag is used to indicate who has control over the model and to ensure that only one participant (the one with the green flag) can modify the model at any one time. If the flag is available, any user (server or client) can take the flag (which triggers a locking mechanism) and manipulate the model. All the changes made to the model can be seen by any other party that is connected to the server. The locking mechanism disables other users' ability to manipulate the model while someone else is modifying the model. However, all changes made can be seen by all the users on their own Tablet PC (Figure 5). In this way synchronous collaboration and coordination is promoted as users do not have to save the model and book it into a repository or email it to a co-worker. The changes can be seen by everyone as they are made and stakeholders can agree or disagree immediately. Once the modeller has finished his/her modelling tasks, the flag can be passed to a different modeller. The current modeller only taps on the green flag and the flag will then go white. All of the connected devices will show a white flag. The first participant to tap on the white flag will then have the right to model and a green flag will appear on that device. The other participants will have a red flag indicating that they have read-only rights.

\subsection{Design of the evaluation process}

In order to determine the usability of a software product, some studies (Nielsen \& Molich, 1990; Virzi, 1992; Nielsen, 2000, 2012) have proposed that five to eight participants are sufficient for a usability evaluation. However, Law and Hvannberg (2004) propose that at least 11 participants are 


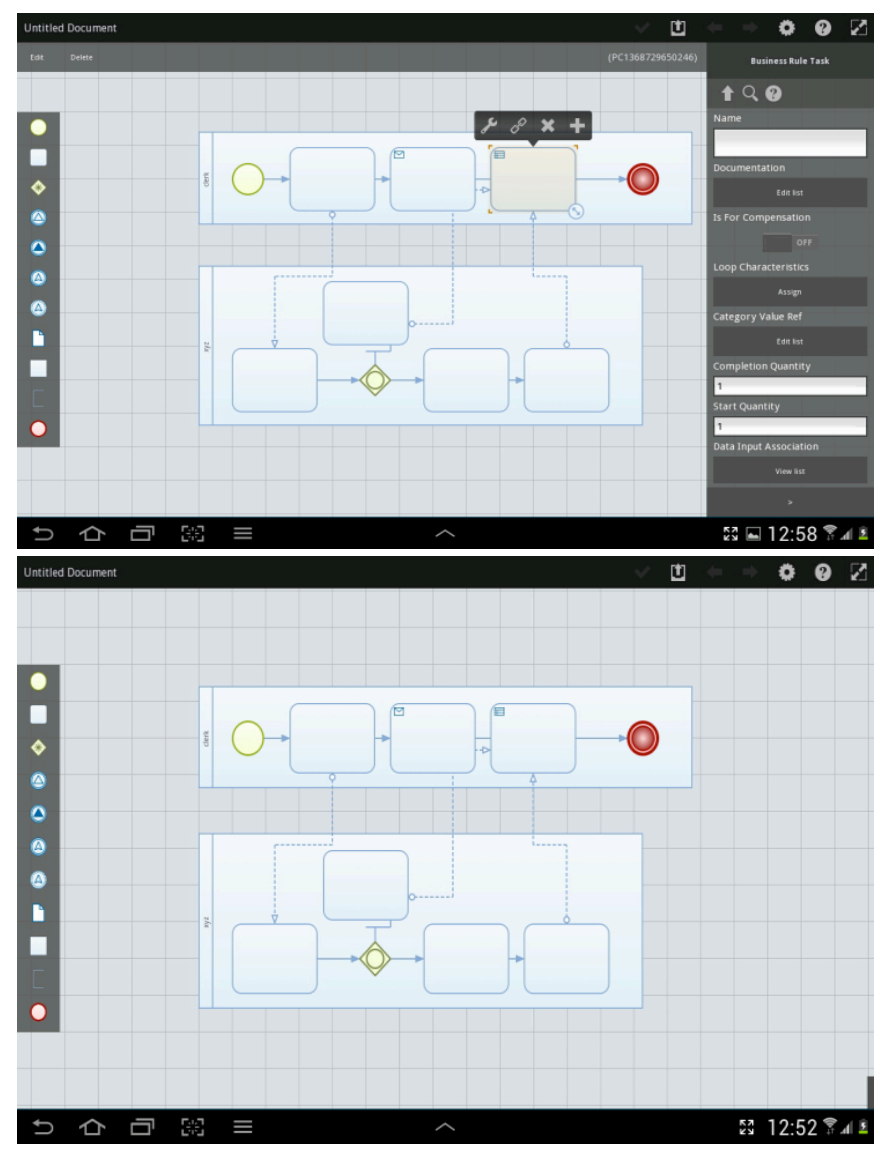

Figure 5: Example of two users working on the same model

required. W. Hwang and Salvendy (2010) also argue that at least $10 \pm 2$ participants are optimal for a usability evaluation in order to predict $80 \%$ of potential usability problems. The target sample for this study was industry BPM modellers and students who do BPM in teams as part of their coursework, therefore modellers from industry and students were invited to take part in the evaluation and the goal sample size was at least 16 participants.

The evaluations had to be carried out in a group environment in order to evaluate the collaboration of the BPM-Touch software tool (Tse, Shen, Greenberg \& Forlines, 2007). Before each evaluation, the reason for the evaluation was explained to the participants; they were required to sign a consent form and were given a short introduction to BPM-Touch as well as to the concepts of business process diagrams. All of the evaluations were video recorded with the participants' consent.

The research instrument used in the evaluation was a post-test questionnaire and consisted of biographical details and a usability section consisting of closed-ended questions and open-ended questions. The closed-ended questions were designed based on the attributes of usability described by ISO (2011) and Nielsen (1994). These attributes are effectiveness, efficiency and satisfaction. Effectiveness was measured by calculating the number of completed, successful tasks compared to 
the unsuccessful tasks (Nielsen, 1994). Efficiency (task time) was measured by recording the time it took each pair of participants to complete the task and compared with a target time.

The questionnaire included three sections related to satisfaction. A 5-point Likert scale rating was used for this section where 1 represents Strongly disagree and 5 represents Strongly agree. The first section consisted of the 19 standard Post Study System Usability Questionnaire (PSSUQ) questions, which is a 19-item instrument designed for assessing a user's perception of their satisfaction with using a system (Lewis, 1995). The PSSUQ consists of three areas, namely: Information Quality (InfoQual), Interface Quality (IntQual) and System Usefulness (SysUse). Collaboration and satisfaction with gesture manipulation was measured by metrics identified in literature (Hornecker et al., 2008; Kammer et al., 2010; Hunter \& Maes, 2008; Sams et al., 2011; Tse et al., 2007). The second and third categories of satisfaction evaluated related to satisfaction with the collaboration and gesture manipulation features of the technology respectively.

Face validity of the questionnaire was established by using questions based on literature and content validity was established by means of a pilot study (Saunders, Lewis \& Thornhill, 2011, pp. 108145). The pilot study was carried out with a pair of participants to test the software, questionnaire reliability and to ensure that there was no ambiguity in the task list or the questionnaire. No changes were made to the questionnaire after the pilot study.

Creswell's data analysis procedure for qualitative research (2009) was followed for analysing the qualitative data in order to become familiar with the data, the analysis and identifying themes. This analysis incorporates recognising and analysing themes and reporting on the themes found within the data. As part of the qualitative analysis, a check on the reliability for all of the questionnaire responses was carried out constantly by following Gibbs' procedures (2008). The completed questionnaires were reviewed several times by the researcher to ensure that there were no errors, the codes were continuously compared to make sure that the code meanings did not change and a different researcher examined the codes. Validity was also ensured by describing the findings as thoroughly as possible, avoiding and removing any possible bias and noting negative behaviour (Creswell, 2009).

\section{RESULTS AND DISCUSSION}

\subsection{Participant profile}

A total of 18 participants from both industry and education were selected for the BPM-Touch evaluation, in order to represent both sample groups. There were four pairs of BPM students and five pairs of industry participants that took part in the evaluation in a thinking-aloud environment as the partners were allowed to communicate with each other and discuss BPM-Touch as well as the tasks (Table 1). All of the student and industry participants were required to have had some experience with BPM in order to participate in the study. The eight student participants were all between the ages of 18 and 25 (Table 1). Five of the student participants were males and three were females. Fifty percent $(n=4)$ of the students had more than ten years' experience with computers, $38 \%$ $(n=3)$ had between six and ten years' experience and $12 \%(n=1)$ had one to five years' experience with computers. Ten industry participants completed the evaluation of which eight were males 
and two were females. The majority of the industry participants $(n=7)$ were between the ages of 26 and 35. Ninety percent of the industry participants have had more than ten years' experience with computers. The industry participants were selected based on the criteria that they had some modelling experience in either BPM or UML modelling.

Table 1: Participant profile $(n=18)$

\begin{tabular}{|l|l|c|c|}
\hline Variable & Groups & $\begin{array}{c}\text { Industry } \\
n\end{array}$ & $\begin{array}{c}\text { Students } \\
n\end{array}$ \\
\hline \multirow{3}{*}{ Gender } & Male & 8 & 5 \\
& Female & 2 & 3 \\
& Total & $\mathbf{1 0}$ & $\mathbf{8}$ \\
\hline \multirow{3}{*}{ Age } & $18-25$ & 3 & 8 \\
& $26-35$ & 7 & 0 \\
\hline \multirow{3}{*}{ Computer experience } & Total & $\mathbf{1 0}$ & $\mathbf{8}$ \\
& 1-5 years & 0 & 1 \\
& 6-10 years & 1 & 3 \\
& >10 years & 9 & 4 \\
& Total & $\mathbf{1 0}$ & $\mathbf{8}$ \\
\hline
\end{tabular}

\subsection{Effectiveness and efficiency results}

The students were the first to evaluate the software prototype and all four pairs of students decided not to practise using the software and started with the evaluation straight away. The first evaluation was the quickest and was completed in 15 minutes, whilst the second evaluation was completed in 17 minutes (Table 2). The third and fourth evaluations took longer due to some minor teething technical issues and the Tablet PC had to be restarted.

The five pairs of industry participants also decided not to practise using the software and started with the evaluation straight away. The fastest completion time was the second pair who completed the evaluation in 18 minutes, followed by the fourth pair who completed it in 19 minutes. The first group completed the evaluation in 21 minutes and the fifth group in 25 minutes. The third group did not manage to finish all the activities in the task due to technical errors with the Wi-Fi speed.

The BPM-Touch system can be considered effective since $89 \%$ of the pairs (eight out of nine pairs) were able to successfully and accurately complete the task (Donyaee, 2001; Seffah, Donyaee, Kline \& Padda, 2006). The results show that the BPM-Touch system is efficient since all of the participant teams completed the tasks in less time than the goal task time of 40 minutes. The mean times for the students and participants from industry are very similar, based on all completed tasks. 
Table 2: BPM-Touch evaluation with total task time

\begin{tabular}{|c|c|c|}
\hline Team number & Total task time (minutes) & Completion (\%) \\
\hline \multicolumn{3}{|c|}{ Student evaluation } \\
\hline 1 & 15 & 100 \\
2 & 17 & 100 \\
3 & 28 & 100 \\
4 & 22 & 100 \\
\hline Mean time & $\mathbf{2 0 . 5}$ \\
\hline \multicolumn{3}{|c|}{ Industry evaluation } \\
\hline 1 & 21 & 100 \\
3 & 18 & 100 \\
4 & 29 & 90 \\
5 & 19 & 100 \\
Mean time & 25 & 100 \\
\hline
\end{tabular}

\subsection{Satisfaction results}

Satisfaction had three categories, namely PSSUQ (Section 4.3.1), collaboration (Section 4.3.2) and gesture manipulation (Section 4.3.3).

\subsubsection{PSSUQ}

The overall PSSUQ scores for both the industry group $(\mu=3.92)$ and the student group $(\mu=4.12)$ were in the positive range. An analysis of the four categories of PSSUQ (Overall, SysUse, InfoQual and InterQual) revealed that the mean scores for both the student group and the industry group for all categories were also in the positive range (Figure 6). However the students gave a higher mean rating than the industry group for all four categories.

The analysis of the results of the detailed PSSUQ data revealed that the item with the highest mean rating ( $\mu=4.63$ ) in the student sample was for "BPM-Touch was easy to learn" (Table 3). The two items with the highest mean ratings in the industry sample of participants were "The Interface is pleasant" $(\mu=4.40)$ and they "Liked working with the interface" $(\mu=4.40)$. From this it can be deduced that in general, participants were satisfied with the usability of BPM-Touch since in both groups nearly $90 \%$ of items had a mean rating in the positive range.

Only two of the 19 PSSUQ items were not rated in the positive range and these were both in the neutral range. The two items were both related to error handling and were "Clear error messages provided" and "Can recover easily from mistakes". 


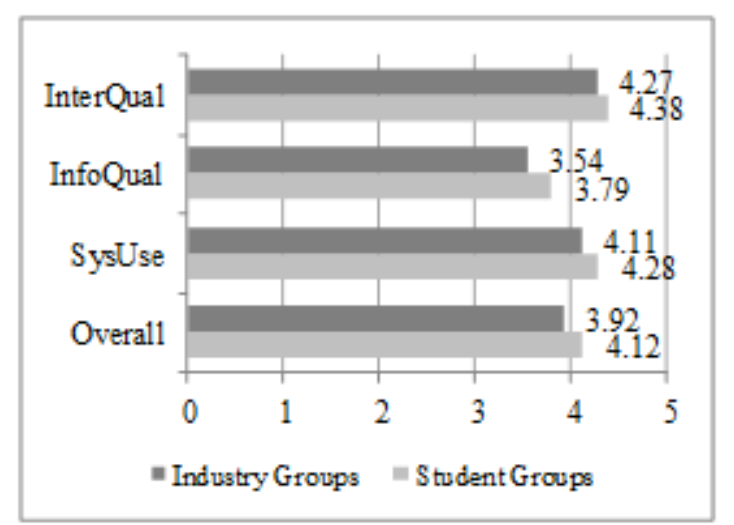

Figure 6: PSSUQ scores $(n=18)$

Table 3: Usability (PSSUQ)

\begin{tabular}{|l|c|c|}
\hline Metric item & Industry & Students \\
\hline Easy to use & 3.90 & 4.25 \\
Simple to use & 4.20 & 4.50 \\
Complete tasks effectively & 4.10 & 4.50 \\
Could complete tasks quickly & 3.90 & 4.00 \\
Complete tasks efficiently & 4.30 & 3.63 \\
Comfortable using & 4.00 & 4.38 \\
Easy to learn & 4.20 & 4.63 \\
Can be productive with this system & 4.30 & 4.38 \\
Clear error messages provided & 2.60 & 2.63 \\
Can recover easily from mistakes & 3.00 & 3.38 \\
Clear information provided & 3.70 & 4.00 \\
Easy to find information & 3.60 & 4.13 \\
Easy to understand information & 3.80 & 4.13 \\
Information effective to help me & 4.10 & 4.25 \\
Clear organisation of information & 4.00 & 4.00 \\
Interface was pleasant & 4.40 & 4.25 \\
Liked using the interface & 4.40 & 4.50 \\
The system has all the capabilities needed & 4.00 & 4.38 \\
I am satisfied with the system & 4.00 & 4.38 \\
\hline Overall PSSUQ score & 3.92 & 4.12 \\
\hline
\end{tabular}


From this it can be deduced that the students and industry participants were neutral towards the error handling features of BPM-Touch. This is to be expected since the experiments were performed on an early prototype of the system and this system had not undergone extensive reliability and error testing. This is possibly an area for improvement in future research.

\subsubsection{Collaboration results}

The SIDV collaboration style (Isenberg et al., 2010) was followed by all of the participants. Figure 7 (a) shows how the two participants in a pair each looked at their own model while the modeller in charge was modelling. In most cases the participants divided the diagram between them so that both participants had a chance to model on BPM-Touch and to experience the software. Figure 7 (b) shows a completed model. When a participant was stuck, the partner would help by either explaining what to do or by showing his/her partner on their Tablet PC. In several cases, one participant read the object labels out loud and the partner would then type in the labels.

After completing the modelling tasks each participant completed the post-task questionnaire. The closed-ended questions were 5-point Likert scale metric items, therefore the results were grouped statistically into three ranges: 'positive', 'neutral' and 'negative': $x$ is negative where $1 \leq x<2.6$; neutral where $2.6 \leq x<3.4$; and positive where $3.4 \leq x<5$.

For the collaboration section of the questionnaire, all of the six metric items had mean ratings in the positive range (Table 4). The item "My experience with CBPM in this exercise has been positive", was rated positively by both the industry $(\mu=4.40)$ and the student group $(\mu=4.50)$. The item "I can easily share ideas using this system" is the only item that received a mean value of less than four from both students and industry; however both ratings are still positive.

Table 4: Collaboration results

\begin{tabular}{|l|c|c|}
\hline Metric item & Industry & Students \\
\hline Minimal mistakes were made due to collaborating & 4.30 & 3.88 \\
with other team members. & & \\
My experience with CBPM in this exercise has been & 4.40 & 4.50 \\
positive. & 4.50 & 4.13 \\
I was able to collaborate easily. & 4.20 & 3.63 \\
Having a partner made the task of modelling easier. & 3.90 & 3.88 \\
I can easily share ideas using this system. & 4.60 & 4.38 \\
I was able to easily communicate with my team & & \\
member(s). & 4.37 & $\mathbf{4 . 1 3}$ \\
\hline Overall collaboration mean score & & \\
\hline
\end{tabular}

From the analysis of the results it is evident that the participants were satisfied with the collaboration aspects of BPM-Touch. The results confirm that effective coordination improves the effectiveness of collaborative tasks (Denise, 1999). The benefits of collaborative modelling (Scholtz et al., 2013; 

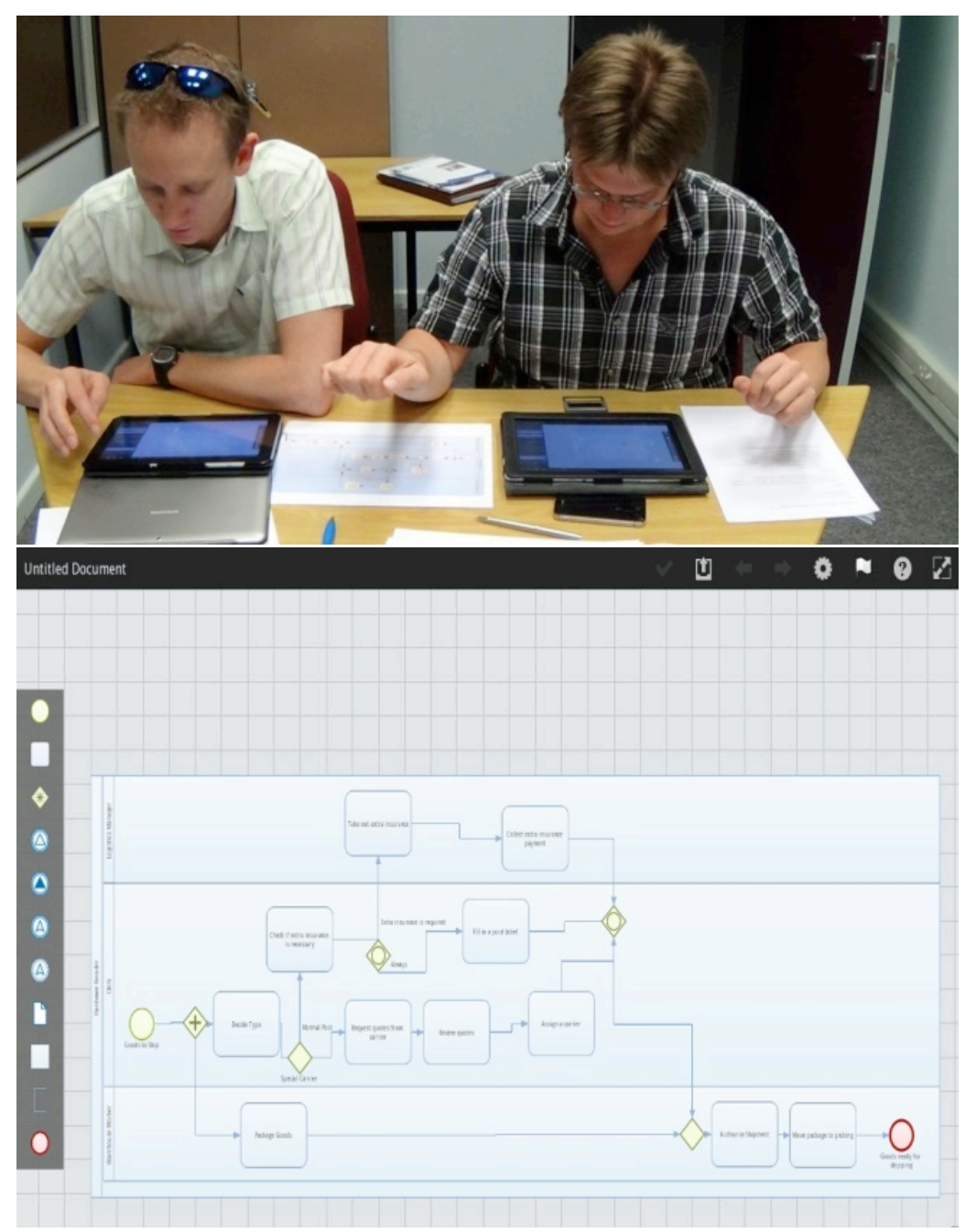

Figure 7: Collaboration by industry participants: (a) participants; (b) a completed model

Forster et al., 2012) as well as the advantages of using Tablet PCs for collaborative tasks (Hinckley, 2003; Hinckley et al., 2004) are also verified by these results.

\subsubsection{Gesture manipulation results}

The gesture manipulation metrics (Figure 8) all had mean positive ratings for both groups of participants. The highest rated metric item by students was "Gestures were logical and easily remembered" ( $\mu=4.63)$ and the highest rated item by industry was "Touch computers made collaboration easier" ( $\mu=4.50)$. The lowest rated item from students ( $\mu=4.00)$, and industry ( $\mu=4.13$ ), was "BPM-Touch correctly interpreted the gestures" but they were both still in the positive range. 


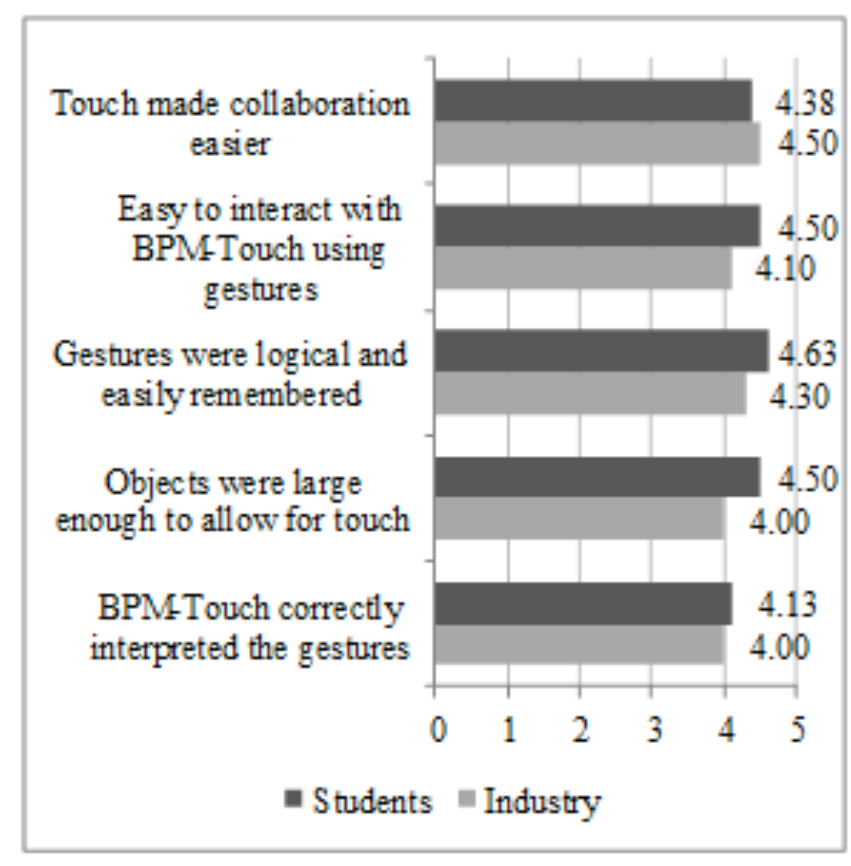

Figure 8: Gesture manipulation

The participants were asked to list any positive aspects relating to the use of BPM-Touch. The majority (80\%) of industry participants indicated that the collaborative functionality is a positive aspect of BPM-Touch. As one participant stated, "it works well for collaborative purposes". Participants were also asked to list any challenges that they had with using BPM-Touch. The challenges reported were that the keyboard was sometimes difficult to use and that the device was slow at times. Two students indicated that the connection between the Tablet PCs was faulty at times. The reason for the connection failure or slow integration could be due to slow Wi-Fi speeds encountered while using BPM-Touch. Two of the industry participants stated that they would have liked an undo function in BPM-Touch as they had to delete objects and redo the function.

Using the video recordings and observations made by the author during the evaluation sessions it was evident that all the participants collaborated with their partners. They collaborated by working together and by discussing the model and who needed to do what. The participants also helped each other when errors occurred and most of the industry participants coordinated constantly by changing control (flag) to their partners several times throughout the evaluation. An analysis of the video observations confirmed the quantitative and qualitative results of the post-task questionnaire that the participants enjoyed several benefits of CBPM and overcame several challenges of CBPM by using the BPM-Touch prototype. 


\section{CONCLUSIONS}

This paper has proposed an approach to coordination using synchronous gesture manipulation input by means of the development of a prototype (BPM-Touch) and an evaluation of the prototype. Several collaboration, usability and gesture manipulation metrics were identified in literature and used to evaluate the prototype and the results were all positive. The results showed that the BPM-Touch prototype supports collaboration by displaying updated, real-time information on all of the connected devices. BPM-Touch can be used by multiple users synchronously in a co-located environment and was rated as effective and efficient. Participants also rated the BPM-Touch system as easy to use. This study has therefore successfully answered the research question "Can synchronous gesture manipulation be used for the effective collaboration and coordination of BPM tasks?".

This paper provides a valuable contribution to the research field of collaborative work, particularly with regards to the SIDV approach using gesture manipulation. Users can make use of this approach and the proposed BPM-Touch software for the collaboration and coordination of business process modelling. Researchers can use the results of this study to better understand the way people collaborate and coordinate tasks using gesture manipulation. Whilst the study was limited by a small sample size, it was sufficient for a usability study. Further research could perform larger studies on the reliability of the BPM-touch approach and could extend the study to dispersed environments.

\section{References}

Apted, T. \& Kay, J. (2008). PhoTable: Enhancing the social interaction around the sharing of digital photographs. In Proceedings of CHI 08 Workshop on Collocated Social Practices Surrounding Photos. Citeseer.

Bamforth, R. (2012). Tablets in the enterprise: Challenges of office mobility. Last accessed 20 Nov 2015. Retrieved from http://www.computerweekly.com/feature/Challenges-of-officemobility

Bandara, W., Chand, D. R., Chircu, A. M., Hintringer, S., Karagiannis, D., Recker, J. C., ... Welke, R. J. (2010). Business process management education in academia: Status, challenges, and recommendations. Communications of the Association for Information Systems, 27, 743-776.

Barjis, J. (2011). CPI modeling: Collaborative, participative, interactive modeling. In Proceedings of the Winter Simulation Conference (pp. 3099-3108). http://dx.doi.org/10.1109/wsc.2011. 6148009

Berry, M. \& Hamilton, M. (2006). Mobile computing, visual diaries, learning and communication: Changes to the communicative ecology of design students through mobile computing. In Proceedings of the 8th Australasian Conference on Computing Education (Vol. 52, pp. 35-44). Australian Computer Society, Inc.

Bonastre, O. M., Benavent, A. P. \& Belmonte, F. N. (2006). Pedagogical use of tablet PC for active and collaborative learning. In Proceedings of the 2006 IEEE International Professional Communication Conference (pp. 214-218). IEEE. http://dx.doi.org/10.1109/ipcc.2006.320350 
Clifton, P., Mazalek, A., Sanford, J., Rébola, C., Lee, S. \& Powell, N. (2011). SketchTop: Design collaboration on a multi-touch tabletop. In Proceedings of the Fifth International Conference on Tangible, Embedded, and Embodied Interaction (pp. 333-336). ACM. http://dx.doi.org/10. $1145 / 1935701.1935778$

Creswell, J. W. (2009). Research design: Qualitative, quantitative, and mixed methods approaches (3rd). Sage.

Denise, L. (1999). Collaboration vs. c-three (cooperation, coordination, and communication). Innovating, 7(3), 1-6.

Derizemlya, Y. (2009). Microsoft Surface: Advantages and disadvantages. Last accessed 23 Oct 2013. Retrieved from http://yderizem.blogspot.com/2009/10/google-chrome-advantagesand.html

Dollmann, T., Houy, C., Fettke, P. \& Loos, P. (2011). Collaborative business process modeling with CoMoMod-A toolkit for model integration in distributed cooperation environments. In Proceedings of the 20th IEEE International Workshops on Enabling Technologies: Infrastructure for Collaborative Enterprises (WETICE), 2011 (pp. 217-222). http://dx.doi.org/10.1109/wetice. 2011.36

Donyaee, M. K. (2001). Towards an integrated model for specifying and measuring quality in use (Master's thesis, Concordia University).

Ellis, C. A., Gibbs, S. J. \& Rein, G. (1991). Groupware: Some issues and experiences. Communications of the ACM, 34(1), 39-58. http://dx.doi.org/10.1145/99977.99987

Flügel, B., Seymour, L. \& van der Merwe, F. (2014). Challenges to business process teaching by South African information systems lecturers. In Proceedings of the South African Computer Lecturers' Association (SACLA) 2014 (pp. 25-26).

Forster, S., Pinggera, J. \& Weber, B. (2012). Collaborative business process modeling. In Proceedings of EMISA '12 (Vol. 206, pp. 81-94).

Gaskell, A. (2012, April). Cloud technologies fuel growth in BPM market. Retrieved from http: //www.processexcellencenetwork.com/business-process-management-bpm/articles/ cloud-technologies-fuel-growth-in-bpm-market/

Gibbs, G. R. (2008). Analysing qualitative data. Sage.

Hevner, A. R. (2007). A three cycle view of design science research. Scandinavian Journal of Information Systems, 19(2), 4.

Hevner, A. R., March, S. T., Park, J. \& Ram, S. (2004). Design science in information systems research. MIS Quarterly, 28(1), 75-105.

Hinckley, K. (2003). Synchronous gestures for multiple persons and computers. In Proceedings of the 16th Annual ACM Symposium on User Interface Software and Technology (pp. 149-158). ACM. http://dx.doi.org/10.1145/964696.964713

Hinckley, K., Ramos, G., Guimbretiere, F., Baudisch, P. \& Smith, M. (2004). Stitching: Pen gestures that span multiple displays. In Proceedings of the Working Conference on Advanced Visual Interfaces (pp. 23-31). ACM. http://dx.doi.org/10.1145/989863.989866

Hornecker, E., Marshall, P., Dalton, N. S. \& Rogers, Y. (2008). Collaboration and interference: Awareness with mice or touch input. In Proceedings of the 2008 ACM Conference on Computer- 
Supported Cooperative Work (pp. 167-176). ACM. http://dx.doi.org/10.1145/1460563. 1460589

Hunter, S. \& Maes, P. (2008). WordPlay: A table-top interface for collaborative brainstorming and decision making. In Proceedings of the Third IEEE International Workshop on Tabletops and Interactive Surfaces (pp. 2-5). Citeseer.

Hwang, G.-J., Wu, C.-H. \& Kuo, F.-R. (2013). Effects of touch technology-based concept mapping on students' learning attitudes and perceptions. Journal of Educational Technology \& Society, 16(3), 274-285.

Hwang, W. \& Salvendy, G. (2010). Number of people required for usability evaluation: The $10 \pm 2$ rule. Communications of the ACM, 53(5), 130-133. http://dx.doi.org/10.1145/1735223. 1735255

Isenberg, P., Fisher, D., Morris, M. R., Inkpen, K. \& Czerwinski, M. (2010). An exploratory study of co-located collaborative visual analytics around a tabletop display. In Proceedings of the IEEE Symposium on Visual, Analytics, Science and Technology (VAST) 2010 (pp. 179-186). IEEE. http://dx.doi.org/10.1109/VAST.2010.5652880

ISO. (2011). ISO/IEC 25010:2011 systems and software engineering-Systems and software quality requirements and evaluation (square)-System and software quality models. ISO/IEC.

Johannesson, P. \& Perjons, E. (2012). A design science primer. Lexington: CreateSpace.

Kammer, D., Wojdziak, J., Keck, M., Groh, R. \& Taranko, S. (2010). Towards a formalization of multi-touch gestures. In Proceedings of the ACM International Conference on Interactive Tabletops and Surfaces (pp. 49-58). ACM. http://dx.doi.org/10.1145/1936652.1936662

Ko, R. K., Lee, S. S. \& Wah Lee, E. (2009). Business process management (bpm) standards: A survey. Business Process Management Journal, 15(5), 744-791. http://dx.doi.org/10.1108/ 14637150910987937

Law, E. L.-C. \& Hvannberg, E. T. (2004). Analysis of combinatorial user effect in international usability tests. In Proceedings of the SIGCHI Conference on Human Factors in Computing Systems (pp. 916). ACM. http://dx.doi.org/10.1145/985692.985694

Lewis, J. R. (1995). IBM computer usability satisfaction questionnaires: Psychometric evaluation and instructions for use. International Journal of Human-Computer Interaction, 7(1), 57-78. http://dx.doi.org/10.1080/10447319509526110

Mendling, J. (2007). Detection and prediction of errors in EPC business process models (Doctoral dissertation, Wirtschaftsuniversität Wien Vienna).

Morris, M. R., Huang, A., Paepcke, A. \& Winograd, T. (2006). Cooperative gestures: Multi-user gestural interactions for co-located groupware. In Proceedings of the SIGCHI Conference on Human Factors in Computing Systems (pp. 1201-1210). ACM. http://dx.doi.org/10.1145/ 1124772.1124952

Nielsen, J. (1994). Usability engineering. Elsevier.

Nielsen, J. (2000). Why you only need to test with 5 users. Accessed 20 Nov 2015. Retrieved from http://www.nngroup.com/articles/why-you-only-need-to-test-with-5-users/

Nielsen, J. (2012). How many test users in a usability study? Accessed 20 Nov 2015. Retrieved from http://www.nngroup.com/articles/how-many-test-users/ 
Nielsen, J. \& Molich, R. (1990). Heuristic evaluation of user interfaces. In Proceedings of the SIGCHI Conference on Human Factors in Computing Systems (pp. 249-256). ACM. http://dx.doi.org/ $10.1145 / 97243.97281$

Object Management Group. (2006). Business process model and notation. Retrieved from http: //www.omg.org/spec/BPMN/

Oleksik, G., Milic-Frayling, N. \& Jones, R. (2014). Touch and gesture: Mediating content display, inscriptions, and gestures across multiple devices. Personal and Ubiquitous Computing, 18(5), 1243-1257. http://dx.doi.org/10.1007/s00779-013-0724-5

PC Tech Staff. (2014). Surging demand for tablets in South Africa results in sicknote for traditional notebook vendors. Retrieved from http://pctechmag.co.za/2014/06/surging-demand-fortablets-in-south-africa-results-in-sicknote-for-traditional-notebook-vendors/

Peffers, K., Tuunanen, T., Rothenberger, M. A. \& Chatterjee, S. (2007). A design science research methodology for information systems research. Journal of Management Information Systems, 24(3), 45-77. http://dx.doi.org/10.2753/MIS0742-1222240302

Recker, J. (2010a). Continued use of process modeling grammars: The impact of individual difference factors. European Journal of Information Systems, 19(1), 76-92. http://dx.doi.org/10.1057/ ejis. 2010.5

Recker, J. (2010b). Opportunities and constraints: The current struggle with BPMN. Business Process Management Journal, 16(1), 181-201. http://dx.doi.org/10.1108/14637151011018001

Renger, M., Kolfschoten, G. L. \& de Vreede, G.-J. (2008). Using interactive whiteboard technology to support collaborative modeling. In Groupware: Design, implementation, and use (pp. 356-363). Springer.

Sams, I., Wesson, J. \& Vogts, D. (2011). An architecture to support multi-touch collaborative information retrieval. In Proceedings of the Southern Africa Telecommunication Networks and Applications Conference (SATNAC) 2011 (pp. 369-374).

Saunders, M., Lewis, P. \& Thornhill, A. (2011). Research methods for business students (5th edition). Pearson Education.

Scheer, A.-W. (1998). Architecture of integrated information systems (ARIS). In Business process engineering study edition: Reference models for industrial enterprises (pp. 4-16). Springer.

Scheer, A.-W. \& Brabänder, E. (2010). The process of business process management. In Handbook on business process management 2 (pp. 239-265). Springer.

Scholtz, B., Calitz, A. \& Snyman, I. (2013). The usability of collaborative tools: Application to business process modelling. In Proceedings of the South African Institute for Computer Scientists and Information Technologists Conference 2013 (pp. 347-358). http:// dx.doi.org/10.1145/ 2513456.2513503

Scott, S. D., Carpendale, M. S. T. \& Inkpen, K. M. (2004). Territoriality in collaborative tabletop workspaces. In Proceedings of the 2004 ACM Conference on Computer-Supported Cooperative Work (pp. 294-303). ACM. http://dx.doi.org/10.1145/1031607.1031655

Seethamraju, R. (2012). Business process management: A missing link in business education. Business Process Management Journal, 18(3), 532-547. http:/ / dx. doi .org / 10.1108/ 14637151211232696 
Seffah, A., Donyaee, M., Kline, R. B. \& Padda, H. K. (2006). Usability measurement and metrics: A consolidated model. Software Quality Journal, 14(2), 159-178. http://dx.doi.org/10.1007/ s11219-006-7600-8

Sheehy, K., Kukulska-Hulme, A., Twining, P., Evans, D., Cook, D. \& Jelfs, A. (2005). Tablet PCs in schools: A review of literature and selected projects. BECTA.

Tabtou. (2012). ProcessCraft-A BPMN 2.0 visual editor. Online. Accessed 17 Jul 2013. Retrieved from http://showgen.com/\# extensive-context-sensitive-help

Technology Evaluation Centers. (2013). TEC 2013 ERP buyer's guide for process manufacturing. Retrieved from http://www.technologyevaluation.com/view_document/report/34885/ tec-2013-erp-buyer-s-guide-for-process-manufacturing.html

Topi, H., Valacich, J. S., Wright, R. T., Kaiser, K., Nunamaker Jr, J. F., Sipior, J. C. \& de Vreede, G.-J. (2010). IS 2010: Curriculum guidelines for undergraduate degree programs in information systems. Communications of the Association for Information Systems, 26(1), 18.

Tse, E., Shen, C., Greenberg, S. \& Forlines, C. (2007). How pairs interact over a multimodal digital table. In Proceedings of the SIGCHI Conference on Human Factors in Computing Systems (pp. 215218). ACM. http://dx.doi.org/10.1145/1240624.1240659

Van Greunen, D., Van Der Merwe, A. \& Kotze, P. (2010). Factors influencing BPM tools: The influence on user experience and user interfaces. International Journal of Computing and ICT Research, 4(1), 47-57.

Virzi, R. A. (1992). Refining the test phase of usability evaluation: How many subjects is enough? Human factors: The Journal of the Human Factors and Ergonomics Society, 34(4), 457-468.

White, S. (2004). Business process modeling notation. Accessed: 25 Sep 2012. Retrieved from http://www.omg.org/bpmn/\%20Documents/BPMN_V1-0_May_3_2004.pdf 